\title{
Viral Suppression in the Setting of Multidrug Resistance to Nelfinavir, Tenofovir and Abacavir: A Case Report
}

\author{
Amy M Cheng ${ }^{1 *}$, Rustin D Crutchley², Meghann L Davis ${ }^{3}$, Carl Mayberry ${ }^{4}$ and Joseph C Gathe Jr. ${ }^{4}$ \\ ${ }^{1}$ Department of Pharmacy Practice Temple University School of Pharmacy, Philadelphia, PA, USA \\ ${ }^{2}$ Department of Pharmacy Practice and Translational Research, University of Houston, College of Pharmacy, Houston, TX, USA \\ ${ }^{3}$ Department of Pharmacy, Houston Methodist Hospital, Houston, TX, USA \\ ${ }^{4}$ Therapeutic Concepts, P.A., Houston, TX, USA
}

\begin{abstract}
One major barrier to achieving the primary goals of antiretroviral therapy is the development of drug resistance mutations to antiretroviral agents which render them less effective. For patients who are treatment experienced with many drug resistance mutations, selection of an effective antiretroviral regimen becomes challenging. We report a case of a 55 year old male on tenofovir, abacavir and nelfinavir with multiple resistance mutations to each of these agents, yet able to maintain viral suppression and high CD4 T cell counts. This patient was subsequently switched to protease inhibitor monotherapy for the management of HIV in the setting of multidrug resistance.
\end{abstract}

Keywords: HIV; Drug resistant mutations; Proviral DNA genotype; Protease inhibitor monotherapy

\section{Introduction}

The primary goals of antiretroviral therapy (ART) in the treatment of HIV-1 infection are to maximally and durably suppress plasma HIV1 RNA and to preserve immunologic function [1]. A major barrier to achieving these goals is development of resistance mutations to antiretroviral (ARV) agents which render them less effective. Drug resistance mutations (DRMs) may cause resistance to a single ARV agent or confer cross-resistance to an entire drug class, potentially limiting future therapeutic options [2].

DRMs may be identified with resistance tests such as HIV1 genotypes, which are recommended for use in patients who are suspected to have virologic failure [1]. A limitation of the standard genotype is that the viral load of a HIV patient must be at least 500 copies $/ \mathrm{mL}$. For patients who have a viral load of less than 500 copies $/ \mathrm{mL}$ or when the viral load is suppressed ( $<20$ copies/mL), a HIV-1 proviral DNA genotype can provide HIV-1 ARV drug resistance data by next generation sequencing. This is in contrast to the standard genotype that only sequences actively replicating plasma HIV-1 RNA [3].

We report here a case of a 55 year old male on ART with multiple documented resistance mutations, yet maintaining high CD4 T-cell counts and viral suppression. We also evaluated the patient's clinical response when switching from his triple ART regimen to PI monotherapy, darunavir/cobicistat (DRV/COBI).

\section{Case Report}

A 55 year old Caucasian male presents to Therapeutic Concepts (a private adult outpatient HIV clinic in Houston, TX, USA) for a 3 month follow-up appointment for management of HIV-1 infection in January 2016. The patient is here to consider a potential change in ART as discussed at an earlier appointment date.

The patient was diagnosed with HIV-1 subtype B infection in 1995 at the age of 35 years, when presenting to the hospital with symptoms of pneumocystis pneumonia with a nadir CD4 T-cell count of 60 cells $/ \mathrm{mm}^{3}$. The patient's first reported viral load was in March 1996 with a HIV-1 RNA of 6,654 copies/mL. At that time, the patient was treated with dual nucleoside reverse transcriptase inhibitor (NRTI) therapy of lamivudine and zidovudine. Because of insufficient virologic suppression, he was then switched to two new NRTIs stavudine and didanosine in combination with a non-nucleoside reverse transcriptase inhibitor (NNRTI) nevirapine. The patient did not achieve virologic suppression with this regimen and was switched again to his current ART regimen of the nelfinavir (NFV) $625 \mathrm{mg} 2$ tablets twice daily, tenofovir disoproxil fumarate (TDF) $300 \mathrm{mg} 1$ tablet once daily, and abacavir (ABC) $300 \mathrm{mg} 1$ tablet twice daily, which he reports to be taking for over 15 years. The patient currently does not take any other concomitant medications regularly, but occasionally uses loperamide as needed for his diarrheal symptoms. He also denies any use of herbal medications.

During the past two years, the patient experienced some low level viremia periodically (Figure 1). Because the patient has had no prior history of any other type of resistance testing and low level viremia, this prompted us to order a HIV-1 proviral DNA genotype during the clinic visit in October 2015 (Figure 2), which showed complete resistance to his current ART regimen of NFV, TDF, and ABC. Upon the follow-up clinic visit in January 2016, the patient's current ART regimen was changed from TDF, $\mathrm{ABC}$ and NFV to a simpler and more tolerable regimen of PI monotherapy DRV $800 \mathrm{mg} / \mathrm{COBI} 150$ mg 1 tablet once daily, based on results from the HIV-1 proviral DNA genotype. After the patient had been on this new ART regimen of DRV/COBI for three months, we obtained a new set of labs showing a decrease in his HIV-1 viral load from 40 copies/mL to an undetectable viral load (Figure 1) (LabCorp, Burlington, NC, USA). All other standard laboratory tests including lipids, serum chemistries and complete blood count were within normal limits. A CCR5 delta 32 mutation genetic test was also ordered during the April 2016 clinic

*Corresponding author: Amy Cheng, Department of Pharmacy Practice Temple University School of Pharmacy, Philadelphia, PA, 3307 N. Broad Street Philadelphia, PA 19140, USA; Tel: 1 (215) 707-6980; Fax: 1 (215) 707-8326; E-mail: amy.cheng@temple.edu

Received March 30, 2017; Accepted April 05, 2017; Published April 12, 2017

Citation: Cheng AM, Crutchley RD, Davis ML, Mayberry C, Gathe Jr JC (2017) Viral Suppression in the Setting of Multidrug Resistance to Nelfinavir, Tenofovir and Abacavir: A Case Report. J AIDS Clin Res 8: 683. doi: 10.4172/2155-6113.1000683

Copyright: @ 2017 Cheng AM, et al. This is an open-access article distributed under the terms of the Creative Commons Attribution License, which permits unrestricted use, distribution, and reproduction in any medium, provided the original author and source are credited. 
Citation: Cheng AM, Crutchley RD, Davis ML, Mayberry C, Gathe Jr JC (2017) Viral Suppression in the Setting of Multidrug Resistance to Nelfinavir, Tenofovir and Abacavir: A Case Report. J AIDS Clin Res 8: 683. doi: 10.4172/2155-6113.1000683

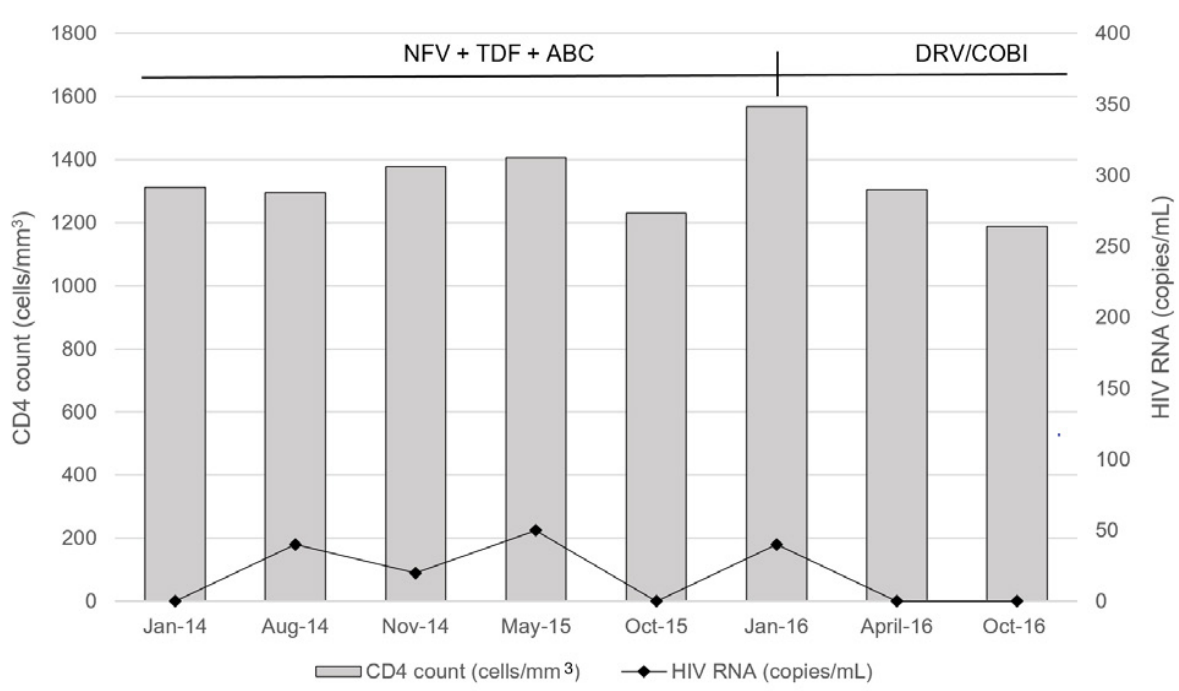

NFV: Nelfinavir; TDF: Tenofovir Disoproxil Fumarate; ABC: Abacavir; DRV/COBI: Darunavir/Cobicistat

Figure 1: HIV viral load and CD4 cell count trends for patient over previous 3 years.

\begin{tabular}{|c|c|c|c|c|}
\hline \multicolumn{2}{|c|}{ Comments } & \multicolumn{3}{|r|}{ HIV-1 Subtype: B } \\
\hline \multirow{8}{*}{$\frac{\bar{\alpha}}{z}$} & $\begin{array}{l}\text { Generic } \\
\text { Name }\end{array}$ & $\begin{array}{l}\text { Brand } \\
\text { Name }\end{array}$ & Assessment & Drug Resistance Associated Mutations Detected \\
\hline & Abacavir & Zisgen & Resistant & M41M/L, M184M/N, L210L/W, T215T/N/S/Y, K219K/N \\
\hline & Didanosine & Videx & Resistant & M41M/L, M184M/V, L210L/W, T215T/N/S/Y, K219K/N \\
\hline & Emtricitabine & Emtriva & Resistant & M41M/L, M184M/V, L210L/W, T215T/N/S/Y, K219K/N \\
\hline & Lamivudine & Epivir & Resistant & M41M/L, M184M/N, L210L/W, T215T/N/S/Y, K219K/N \\
\hline & Stavudine & Zerit & Resistant & M41M/L, L210LW, T215T/N/S/Y, K219K/N \\
\hline & Tenofovir & Viread & Resistant & M41M/L, L210LW, T215T/N/S/Y \\
\hline & Zidovudine & Retrovir & Resistant & M41M/L, L210LW, T215T/N/S/Y, K219K/N \\
\hline \multirow{4}{*}{$\begin{array}{l}\text { E } \\
\frac{\alpha}{Z} \\
Z\end{array}$} & Efavirenz & Sustiva & Resistant & K103KIN, Y181Y/C \\
\hline & Etravirine & Intelence & Resistance Possible & Y181Y/C \\
\hline & Nevirapine & Viramune & Resistant & K103K/N, Y181Y/C \\
\hline & Rilpivirine & Edurant & Resistant & K103K/N, Y181Y/C \\
\hline \multirow{3}{*}{$\bar{\Sigma}$} & Dolutegravir & Trvicay & Sensitive & \\
\hline & Elvitegravir & Vitekta & Sensitive & None \\
\hline & Raltegravir & Isentress & Sensitive & None \\
\hline \multirow{10}{*}{$\bar{\alpha}$} & & Reyataz & Resistance Possible & $\mathrm{D} 30 \mathrm{D} / \mathrm{N}, \mathrm{E} 35 \mathrm{D}, \mathrm{M} 36 \mathrm{M} / \mathrm{I}$ \\
\hline & Atazanavir & Reyataz / $\mathrm{r}$ " & Sensitive & D30D/N, E35D, M36M/I \\
\hline & Darunavir & Prezista $/ \mathrm{r}^{*}$ & Sensitive & None \\
\hline & Fosamprenavir & Lexivg / $\mathrm{r}^{t}$ & Sensitive & E35D \\
\hline & Indinavir & Crixivan $/ \mathrm{r}^{*}$ & Sensitive & M36M/I \\
\hline & Lopinavir & Kaletrat & Sensitive & None \\
\hline & Nelfinavir & Viracept & Resistant & D30D/N, E35D, M36M/I \\
\hline & Ritonavir & Norvir & Sensitive & E35D \\
\hline & Saquinavir & Invirase / $\mathrm{r}^{\mathrm{4}}$ & Sensitive & D30D/N, E35D \\
\hline & Tipranavir & Aptivus / $\mathrm{r}^{\mathrm{t}}$ & Sensitive & E35D, M36M/I \\
\hline
\end{tabular}

Figure 2: HIV-1 proviral DNA genotype results (October 2015).

visit (Quest Diagnostics, San Juan Capistrano, CA, USA) to rule out whether the patient was a long-term non-progressor (LTNP); results were negative for this mutation.

\section{Discussion}

We report the case of an HIV-1 infected patient whose HIV-1 proviral DNA genotype showed resistance to the patient's current regimen, and yet despite this, was able to maintain an undetectable viral load for several years. The patient's HIV-1 proviral DNA genotype showed that he has four thymidine analogue mutations (TAMs) including M41L, L210W, T215Y and K219N, which together decrease susceptibility and cause intermediate to high-level resistance to $\mathrm{ABC}$ 
and TDF. The patient also has the primary mutation D30N, which confers high-level resistance to NFV.

Although the patient showed full resistance to his ART regimen, he was still able to maintain an undetectable viral load with only a few recent "viral blips," defined as "intermittent episodes of detectable lowlevel HIV-1 viremia (viral load between 50-199 copies/mL), which are preceded and followed by an undetectable viral load without any change in therapy" $[1,4]$. Viral blips are a common phenomenon that occurs in about one-quarter of patients who have achieved viral suppression and may be a natural consequence of population dynamics [5]. Because of the patient's high $\mathrm{CD} 4 \mathrm{~T}$-cell counts and low-level viremia, we considered that the patient may have been a LTNP; however, these results were negative.

One potential explanation for continued viral suppression in the setting of a multidrug resistant virus is poor "viral fitness" [6]. It is possible that the patient's resistant virus has a replicative capacity that was lower than that of wild-type virus, especially, since the patient has the M184V mutation which is linked to having a reduced HIV replicative capacity [7]. Another potential explanation could be the apolipoprotein B mRNA-editing, enzyme-catalytic (APOBEC)-induced resistance mutation mechanism which may explain the persistence of the D30N mutation in archived cellular proviral DNA despite viral suppression in some patients [8].

Even though it is uncertain why this patient was able to maintain virologic suppression on his resistant ART regimen, we decided to simplify the patient's ART regimen to an active DRV/COBI monotherapy based off his HIV-1 proviral DNA genotype results. Patients who exhibit characteristics such as high CD4 T-cell counts, sustained viral suppression, and excellent adherence to an ART regimen appear to be the most appropriate candidates for PI monotherapy [9]. This decision was also done intentionally to spare the patient of NRTIs, as he had resistance to the whole class. Our concern for the patient developing drug resistance to DRV/COBI was also low given a recent meta-analysis of PI monotherapy suggesting no increased risk for the development of DRMs [10]. Some studies have demonstrated success with an integrase strand transfer inhibitor (INSTI) plus boosted PI in treatmentexperienced patients $[11,12]$. We decided to not add dolutegravir at this time and potentially use it at a later time in combination with DRV/ COBI should the patient develop persistent low-level viremia.

To date, the patient has been on DRV/COBI for over 12 months and remains clinically stable with a high CD4 T-cell count $\left(1188\right.$ cells $\left./ \mathrm{mm}^{3}\right)$ and viral load suppression to over 48 weeks (Figure 1). According to the patient, the simplification of his ART regimen has been "totally lifechanging" because of his significantly reduced total daily pill burden and improved tolerability. The patient is otherwise healthy and still admits to not taking any other concomitant medications.

\section{Conclusion}

We highlight here a unique patient who has resistance to his prior ART regimen (NFV, $A B C$ and $T D F$ ), yet maintained a suppressed viral load. Potential explanations for this clinical scenario include that the patient might be a LTNP (testing was negative) and poor viral fitness of the patient's resistant viral strain. Another explanation that was not explored is the possibility for drug-gene interactions. NFV is metabolized to its active metabolite nelfinavir hydroxy-t-butylamide (M8) primarily by cytochrome P450 (CYP) isoenzyme 2C19 [13]. Had the patient remained on NFV, we would have considered both pharmacogenetics testing and therapeutic drug monitoring to evaluate the patient's CYP2C19 expression. A poor metabolizer phenotype could lead to significantly higher NFV plasma drug concentrations that could have facilitated his viral suppression even in the setting of highlevel NFV resistance. Given the patient had many DRMs, a phenotype resistance test may have also been helpful to determine the true sensitivities of the patient's HIV-1 virus to his current ART and might have helped confirm the replicative capacity of the patient's HIV-1 virus [1]. However, obtaining results from a phenotype are unlikely since the patient had low-level viremia.

To our knowledge, there have not been any other documented cases of HIV patients maintaining viral suppression with multidrug resistance to their current ART. Ultimately, the patient was successfully simplified to active ART with DRV/COBI monotherapy, maintaining an undetectable viral load on this new regimen, and reporting a much improved quality of life due to considerably decreased pill burden, decreased frequency of administration, and improved tolerability. We learned from this patient that ART is highly individualized and that using HIV-1 proviral DNA genotypes can be advantageous in guiding treatment simplification for treatment experienced patients who are on complex ART regimens, have virologic suppression and/or low-level viremia and have limited information regarding previous resistance testing.

\section{References}

1. Panel on Antiretroviral Guidelines for Adult and Adolescents (2016) Guidelines for the use of antiretroviral agents in HIV-1-infected adults and adolescents.

2. Rhee SY, Taylor J, Fessel WJ, Kaufman D, Towner W, et al. (2010) HIV-1 protease mutations and protease inhibitor cross-resistance. Antimicrob Agents Chemother 54: 4253-4261.

3. Illumina (2016) Understanding the genetic code.

4. Lee PK, Kieffer TL, Siliciano RF, Nettles RE (2006) HIV-1 viral load blips are of limited clinical significance. J Antimicrob Chemother 57: 803-805.

5. Sklar PA, Ward DJ, Baker RK, Wood KC, Gafoor Z, et al. (2002) Prevalence and clinical correlates of HIV viremia ('blips') in patients with previous suppression below the limits of quantification. AIDS 16: 2035-2041.

6. Lucas GM (2005) Antiretroviral adherence, drug resistance, viral fitness and HIV disease progression: A tangled web is woven. J Antimicrob Chemother 55: 413-416.

7. Miller V, Stark T, Loeliger A, Lange J (2002) The impact of the M184V substitution in HIV-1 reverse transcriptase on treatment response. HIV Med 3: $135-145$.

8. Delaugerre C, Braun J, Charreau I, Delarue S, Nere ML, et al. (2012) Comparison of resistance mutation patterns in historical plasma HIV RNA genotypes with those in current proviral HIV DNA genotypes among extensively treated patients with suppressed replication. HIV Med 13: 517-525.

9. Arribas JR, Horban A, Gerstoft J, Fatkenheuer G, Nelson M, et al. (2010) The MONET trial: darunavir/ritonavir with or without nucleoside analogues, for patients with HIV RNA below 50 copies/ml. AIDS 24: 223-230.

10. Arribas JR, Girard P-M, Paton N, Winston A, Marcelin AG, et al. (2016) Efficacy of protease inhibitor monotherapy vs. triple therapy: Meta-analysis of data from 2303 patients in 13 randomized trials. HIV Med 17: 358-367.

11. Latini A, Fabbiani M, Borghi V, Sterrantino G, Giannetti A, et al. (2016) Switching to boosted protease inhibitor plus a second antiretroviral drug (dual therapy) for treatment simplification: A multicenter analysis. BMC Infect Dis 16: 401.

12. Ofotokun I, Sheth AN, Sanford SE, Easley KA, Shenvi N, et al. (2012) A switch in therapy to a reverse transcriptase inhibitor sparing combination of lopinavir/ ritonavir and raltegravir in virologically suppressed HIV-infected patients: A pilot randomized trial to assess efficacy and safety profile: The KITE study. AIDS Res Hum Retroviruses 28: 1196-1206.

13. Burger DM, Schwietert HR, Colbers EPH, Becker M (2006) The effect of the CYP2C $19^{*} 2$ heterozygote genotype on the pharmacokinetics of nelfinavir. $\mathrm{Br} J$ Clin Pharmacol 62: 250-252. 